\title{
Reproducible, high-dimensional imaging in archival human tissue by Multiplexed Ion Beam Imaging by Time-of-Flight (MIBI-TOF)
}

Candace C. Liut, Marc Bosse ${ }^{1 \dagger}$, Alex Kong ${ }^{1}$, Adam Kagel ${ }^{1}$, Robert Kinders², Stephen M. Hewitt $^{3}$, Sean C. Bendall ${ }^{*}$, Michael Angelo ${ }^{1^{*}}$

${ }^{1}$ Department of Pathology, Stanford University, Stanford, California 94304

${ }^{2}$ Office of the Director, Division of Cancer Treatment and Diagnosis, National Cancer Institute, Bethesda, MD 20892

${ }^{3}$ Experimental Pathology Laboratory, Laboratory of Pathology, Center for Cancer Research, National Cancer Institute, National Institutes of Health, Bethesda, MD 20892

${ }^{\dagger}$ Authors contributed equally to this work

${ }^{\star}$ Corresponding Author

\section{Abstract}

Multiplexed ion beam imaging by time-of-flight (MIBI-TOF) is a form of mass spectrometry imaging that uses metal labeled antibodies and secondary ion mass spectrometry to image dozens of proteins simultaneously in the same tissue section. Working with the National Cancer Institute's (NCl) Cancer Immune Monitoring and Analysis Centers (CIMAC), we undertook a validation study, assessing concordance across a dozen serial sections of a tissue microarray of 21 samples that were independently processed and imaged by MIBI-TOF or single-plex immunohistochemistry (IHC) over 12 days. Pixel-level features were highly concordant across all 16 targets assessed in both staining intensity $\left(R^{2}=0.94 \pm 0.04\right)$ and frequency $\left(R^{2}=0.95 \pm 0.04\right)$. Comparison to digitized, single-plex IHC on adjacent serial sections revealed similar concordance $\left(R^{2}=0.85 \pm 0.08\right)$ as well. Lastly, automated segmentation and clustering of eight cell populations found that cell frequencies between replicates yielded an average correlation of $R^{2}=0.92 \pm 0.06$. Taken together, we demonstrate that MIBI-TOF, with well-vetted reagents and automated analysis, can generate consistent and quantitative annotations of clinically relevant cell states in archival human tissue, and more broadly, present a scalable framework for benchmarking multiplexed IHC approaches. 


\section{Introduction}

Immunohistochemistry $(\mathrm{IHC})$ is commonly used in clinical diagnostics and basic research to visualize proteins in intact tissue using chromogenic or fluorescent reporters. ${ }^{1-3} \mathrm{IHC}$ staining is used routinely to guide diagnoses and therapeutic selection in the vast majority of solid tissue malignancies. ${ }^{4-6}$ Although it remains an indispensable tool in anatomic pathology, chromogenic IHC has inherent limitations that hinder quantitative interpretation and prevent routine multiplexed staining. ${ }^{1,7} 8-10$ These drawbacks are particularly limiting in the field of cancer immunotherapy, where accurate evaluation of the tumor immune microenvironment requires the simultaneous mapping of dozens of proteins. ${ }^{11,12}$ The emerging field of spatial-omics with a multitude of analytical solutions is working towards replacement of conventional IHC-driven decision making. ${ }^{11,13}$ While it is clear that new technologies and assays have the potential to generate new types of data, it is unclear whether they can reliably replicate the decision-making information in current 'gold standard' assays.

Our lab has developed multiplexed ion beam imaging by time-of-flight (MIBI-TOF), which avoids the limitations of optical imaging by using secondary mass spectrometry to image dozens of proteins on the same tissue section. ${ }^{14,15}$ In the place of chromogenic or fluorescent reporters, MIBI-TOF uses primary antibodies that are labeled with isotopically enriched metal reporters that can be cleanly delineated and quantified using time-of-flight (TOF) mass spectrometry. Tissue sections are treated with all metal labeled primary antibodies simultaneously using a simple

53 protocol that does not include secondary antibodies, enzymatic amplification, or cyclical staining.

54 During MIBI-TOF analysis, the tissue is sputtered by a primary ion beam in a pixel-by-pixel fashion

55 that liberates the metal tags as secondary ions that are subsequently quantified by TOF. Our lab routinely quantifies 40 targets simultaneously and are currently working towards increasing this

57 capability to 60 or more. ${ }^{16-18}$ Notably, MIBI-TOF is compatible with formalin-fixed, paraffin- 
For technologies like MIBI-TOF to be used in large translational studies and ultimately for

61 routine clinical diagnostics, robustness and reproducibility studies are needed, as are

62 standardized workflows for interpreting these complex data. Furthermore, it is important to show

63 that new technologies are concordant with existing, established clinical assays. In collaboration

64 with the National Cancer Institute $(\mathrm{NCl})$ as part of the Cancer Immune Monitoring and Analysis

65 Centers-Cancer Immunologic Data Commons (CIMAC-CIDC) network ${ }^{22}$, we benchmarked the

66 reproducibility of multiplexed antibody staining using MIBI-TOF. To achieve this, we compared

67 MIBI-TOF imaging data across six independent replicates of adjacent tissue microarray (TMA)

68 serial sections. Antibody staining and MIBI-TOF imaging of each serial section were carried out

69 independently and randomized with respect to all experimental parameters. Additionally, we

70 assessed MIBI-TOF concordance with single-plex IHC chromogenic staining. In keeping with the

71 goal of CIMAC-CIDC to develop comprehensive, standardized immune monitoring analysis for

72 biomarker discovery, we show that MIBI-TOF is a highly reproducible assay and concordant with

73 single-plex IHC.

75 Materials and Methods

\section{Tissue microarray construction and sectioning}

A tissue microarray (TMA) was constructed using human FFPE blocks from Stanford

78 Pathology. The TMA consisted of disease-free controls as well as multiple types of carcinomas,

79 sarcomas, and central nervous system lesions. A table of the cores included in this study can be

80 found in Table 1 ( $1 \mathrm{~mm}$ cores). For each TMA tissue block, 13 consecutive serial sections (4 $\mu \mathrm{m}$

81 section thickness) were acquired. The IHC and MIBI-TOF recuts were alternated so that for the

$82 \mathrm{IHC}$ concordance analysis, MIBI-TOF and IHC could be compared in adjacent sections. The

83 assays performed on each section were as follows:

84 Section 1: H\&E

85 Sections 2,4,6,8,10: Chromogenic IHC 
87 Section 12: MIBI-TOF unstained control

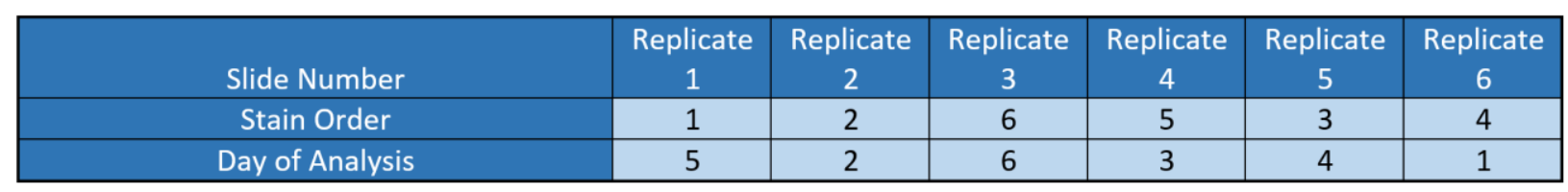

\begin{tabular}{|c|c|c|c|c|c|c|}
\hline FOV & \multicolumn{6}{|c|}{ Order of Acquisition } \\
\hline CONTROL TONSIL & 14 & 4 & 17 & 1 & 19 & 1 \\
\hline CONTROL TONSIL & 18 & 20 & 21 & 7 & 20 & 2 \\
\hline CONTROL TONSIL & 19 & 10 & 6 & 5 & 13 & 3 \\
\hline CONTROL TONSIL & 11 & 1 & 2 & 18 & 5 & 4 \\
\hline CONTROL LYMPH NODE & 9 & 3 & 18 & 2 & 11 & 5 \\
\hline CONTROL LYMPH NODE & 15 & 6 & 11 & 12 & 10 & 6 \\
\hline CONTROL LYMPH NODE & 3 & 7 & 15 & 9 & 4 & 7 \\
\hline CONTROL THYMUS & 7 & 11 & 14 & 20 & 3 & 8 \\
\hline CONTROL THYMUS & 5 & 12 & 5 & 13 & 8 & 9 \\
\hline CONTROL SPLEEN & 17 & 19 & 16 & 16 & 21 & 10 \\
\hline SALIVARY WARTHIN SALIVARY & 2 & 17 & 4 & 4 & 9 & 11 \\
\hline HIGH GRADE, CARCINOMA BLADDER & 16 & 15 & 12 & 19 & 18 & 12 \\
\hline CONTROL PLACENTA & 8 & 9 & 20 & 14 & 7 & 13 \\
\hline DUCTAL CARCINOMA BREAST & 20 & 18 & 3 & 21 & 1 & 14 \\
\hline DUCTAL CARCINOMA BREAST & 1 & 2 & 13 & 15 & 12 & 15 \\
\hline LEIOMYOSARCOMA, MET SOFT TISSUE & 12 & 13 & 7 & 8 & 6 & 16 \\
\hline $\begin{array}{l}\text { HIGH-GRADE MYXOFIBROSARCOMA SOFT } \\
\text { TISSUE }\end{array}$ & 6 & 16 & 19 & 10 & 15 & 17 \\
\hline FOREIGN BODY GIANT CELLS SOFT TISSUE & 21 & 21 & 9 & 6 & 14 & 18 \\
\hline ADENOCARCINOMA COLON & 10 & 8 & 1 & 3 & 2 & 19 \\
\hline PLEOMORPHIC DERMAL SARCOMA SKIN & 13 & 14 & 10 & 11 & 16 & 20 \\
\hline SQUAMOUS CELL CARCINOMA SKIN & 4 & 5 & 8 & 17 & 17 & 21 \\
\hline
\end{tabular}

Table 1: Description of tissue cores and parameter randomization

\section{Antibody preparation}

The full MIBI-TOF panel containing 16 antibodies can be found in Table 2 . The lyophilized

91 antibody panel was obtained from lonpath ("Cell Classification" human panel). Individual metal

92 labeled antibodies for IHC were also obtained from lonpath: CD3 (D7A6E), CD8 (C8/144B), CD68

93 (D4B9C), Pax5 (D7H5X), PanCK (AE1/AE3). Prior to staining, the lyophilized antibody panel was

94 suspended in antibody diluent buffer (TBS-IHC tween, Donkey serum 3\%) and filtered with a 0.1

$95 \mu \mathrm{m}$ filter (Millipore). 


\begin{tabular}{|c|c|}
\hline Target & Clone \\
\hline dsDNA & 3519 DNA \\
\hline beta-tubulin & D3U1W \\
\hline CD4 & EPR6855 \\
\hline CD11c & EP1347Y \\
\hline CD56 & MRQ-42 \\
\hline CD31 & EP3095 \\
\hline CD68 & D4B9C \\
\hline CD8 & C8/144B \\
\hline CD3 & D7A6E \\
\hline Vimentin & D21H3 \\
\hline CD20 & L26 \\
\hline HLA DR & EPR3692 \\
\hline CD45 & 2B11 \& PD7/26 \\
\hline Na-K-ATPase alpha 1 & D4Y7E \\
\hline Pax5 & D7H5X \\
\hline PanCK & AE1/AE3 \\
\hline
\end{tabular}

Table 2: MIBI-TOF staining panel. Targets used for IHC concordance analysis are highlighted in green.

\section{Tissue staining}

Tissue staining for the six MIBI-TOF recuts was carried out over six separate days, randomized with respect to serial section number (Table 1). Detailed protocols can be found here: dx.doi.org/10.17504/protocols.io.byzrpx56, dx.doi.org/10.17504/protocols.io.bf6ajrae, dx.doi.org/10.17504/protocols.io.bhmej43e. We used the Sequenza manual staining system for both MIBI-TOF and IHC staining (dx.doi.org/10.17504/protocols.io.bmc6k2ze).

Briefly, slides were baked at $70^{\circ} \mathrm{C}$ overnight followed by deparaffinization and rehydration with successive washes of 30 seconds and 3 dips each in xylene (3 washes), 100\% ethanol (2 washes), $95 \%$ ethanol (2 washes), $80 \%$ ethanol (1 wash), $70 \%$ ethanol (1 wash), and ddH2O (2 washes) with a Leica ST4020 Linear Stainer (Leica Biosystems). Tissues next underwent antigen retrieval by submerging slides in 3-in-1 Target Retrieval Solution ( $\mathrm{pH} 9$, DAKO Agilent) and incubating at $97^{\circ} \mathrm{C}$ for 40 minutes in a Lab Vision PT Module (Thermo Fisher Scientific). After cooling to room temperature, slides were assembled with a cover plate on a Sequenza rack and washed with PBS wash buffer. The tissue was blocked for 1 hour at room temperature with $1 \mathrm{x}$ TBS IHC Wash Buffer with Tween 20 with 3\% (v/v) normal donkey serum (Sigma-Aldrich), 0.1\% 
$111(\mathrm{v} / \mathrm{v})$ cold fish skin gelatin (Sigma Aldrich), 0.1\% (v/v) Triton X-100, and 0.05\% (v/v) sodium azide.

112 The blocking buffer was washed by adding $200 \mu \mathrm{L}$ of antibody diluent buffer in the Sequenza 113 upper chamber. After the diluent buffer flow through, $120 \mu \mathrm{L}$ of the suspended panel of antibodies 114 was added to the slides. The Sequenza rack was then placed at $4^{\circ} \mathrm{C}$ overnight $(16 \mathrm{hr})$.

115 Following the overnight incubation, slides were washed twice with $1 \mathrm{~mL}$ of PBS wash

116 buffer and fixed in a solution of $2 \%$ glutaraldehyde (Electron Microscopy Sciences) solution in 117 low-barium PBS for 5 minutes. Slides were successively washed with 30 seconds and 3 dips per 118 wash in PBS (1 wash), $0.1 \mathrm{M}$ Tris at $\mathrm{pH} 8.5$ (3 washes), ddH2O (2 washes), and then dehydrated 119 by washing in $70 \%$ ethanol ( 1 wash), $80 \%$ ethanol ( 1 wash), $95 \%$ ethanol ( 2 washes), and $100 \%$ 120 ethanol (2 washes). Slides were dried under vacuum prior to imaging.

121 Single-plex chromogenic IHC was performed for five targets using the same antibody 122 clone as used in the MIBI-TOF staining panel (Table 2). Dewaxing, epitope retrieval, blocking, 123 hybridization, and washing for IHC tissue sections were identical to that of MIBI, with the addition 124 of blocking endogenous peroxidase activity with $3 \% \mathrm{H}_{2} \mathrm{O}_{2}$ (Sigma Aldrich) in ddH2O after epitope 125 retrieval. After overnight primary antibody staining, tissues were washed twice with $1 \mathrm{~mL}$ wash 126 buffer and the antigen:antibody complex detected with the ImmPRESS universal (Anti127 Mouse/Anti-Rabbit) kit (Vector labs). Chromogenic solution (DAB) was incubated for $40 \mathrm{~s}$ and the 128 reaction was stopped with PBS. The slides were counterstained with hematoxylin. $\mathrm{H} \& \mathrm{E}$ tissue sections were reviewed to identify missing tissue cores, cores with large areas 130 of necrosis, tissue folding, and any other macroscopic defect. All IHC and MIBI-TOF data were 131 manually reviewed for excessive background staining.

\section{MIBI-TOF imaging}

The order of image acquisition for each recut was randomized with respect to section

134 number and carried out on six separate days (Table 1). Within each run for each recut, the order 135 of acquisition for the TMA cores was randomized as well (Table 1). Area normalized Xe+ primary 136 ion dose of $9 n A^{*} \mathrm{hr}^{*} \mathrm{~mm}^{-2}$ was used for all MIBI-TOF image acquisitions. At the end of each 
137 imaging run, sensitivity and mass resolution were quantified using a molybdenum foil standard

138 (Supplementary Figure 1).

139 Image processing

MIBI-TOF data processing was performed using a standardized pipeline from lonpath.

141 During data extraction, to only capture the monoatomic peaks that correspond to the monoatomic

142 isotopic labels and avoid the polyatomic ions containing hydrogen, we integrated all peaks using

143 a mass window of $(X-0.3, X)$ where $X$ is the nominal mass-to-charge $(\mathrm{m} / \mathrm{z})$ for each metal reporter

144 tag.

$145 \quad$ Background signal arising from bare slide was removed as described previously using

146 predefined thresholds for $\mathrm{Ba}, \mathrm{Ta}$, and $\mathrm{Au} .{ }^{16,23}$ Isobaric interferences arising from diatomic reporter

147 adducts and polyatomic hydrocarbons were removed iteratively using empirically defined

148 compensation coefficients (Supplementary Table 1). Lastly, we used a combination of several

149 density metrics, including reachability density, connectivity, and Voronoi tessellation to estimate

150 and remove noise from each channel (Supplementary Table 2). These density metrics leverage

151 both spatial and intensity information.

152 Hematoxylin counterstained IHC slides were digitized with the NanoZoomer slide scanner

153 at 40x magnification (Hamamatsu). The threshold for hematoxylin and each DAB channel was

154 set collectively for all images in the staining batch using a DAB channel extracted from the original

155 counterstained image via spectral unmixing. An initial threshold approximation was set with

156 automatic image histogram thresholding. The thresholds were then confirmed by three trained

157 users on controls and additional images for validation and adjusted until consensus was reached

158 (Supplementary Table 3).

159 Marker quantitation

160 In all MIBI-TOF images, for each marker, the frequency and mean intensity of positive

161 pixels in each tissue core was quantified. The resultant value for percent positive pixels (PPP)

162 and mean pixel intensity (MPI) for these images was then normalized with respect to the signal 
163 intensity of endogenous carbon-containing mass peaks originating from the tissue itself. Peaks at

16436,37 and $38 \mathrm{~m} / \mathrm{z}$ corresponding to $3 \mathrm{C}, 3 \mathrm{C} 1 \mathrm{H}$, and $3 \mathrm{C} 2 \mathrm{H}$ were integrated for each image (referred

165 to here as $C_{i}$ ) and the average of these values across all images in all six TMAs was calculated

166 (referred here to $C_{a v g}$ ). To calculate the normalization coefficient $\left(C_{N}\right)$ for a given tissue core, $C_{i}$

167 for that tissue core was divided by $C_{a v g}\left(C_{N}=C_{i} / C_{\text {avg }}\right)$. Median and standard deviation for $C_{N}$

168 across all tissue cores was $1.03 \pm 0.3$. For IHC images, the frequency of positive pixels was

169 quantified using deconvolved and thresholded DAB images (PPP).

170 Reproducibility and concordance

171 To assess replicate reproducibility, least squares linear regression was used to compare

172 PPP for tissue cores in each TMA to the average PPP across all six TMAs. Similarly, least squares

173 linear regression was used to compare MPI for tissue cores in each TMA to the average MPI

174 across all six TMAs. For each comparison, we calculated the slope $(m)$ and coefficient of

175 determination $\left(R^{2}\right)$. To assess concordance of MIBI-TOF with single-plex IHC, we used least

176 squares linear regression to compare values for PPP attained by MIBI-TOF and IHC in adjacent

177 serial tissue sections.

178 Cell segmentation and phenotyping

179 To delineate the location of single cells in MIBI-TOF images, we performed cell 180 segmentation using the pre-trained Mesmer convolutional neural network architecture. ${ }^{24}$ We used 181 dsDNA as the nuclear marker and HLA class I and Na-K-ATPase as the membrane markers as 182 input to the network. The output of Mesmer is the location of each cell in the image.

After cell segmentation, the next step of the analysis pipeline was to determine the

184 phenotype of each individual cell. Pre-processed MIBI-TOF images were first Gaussian blurred 185 using a standard deviation of 2 for the Gaussian kernel. Pixels were normalized by their total 186 expression, such that the total expression of each pixel was equal to 1 . A $99.9 \%$ normalization 187 was applied for each marker. Pixels were clustered into 100 clusters using FlowSOM ${ }^{25}$ based on 188 the expression of 12 phenotypic markers: CD3, CD4, CD8, CD11c, CD20, CD31, CD45, CD56, 
CD68, PanCK, Pax5, and Vimentin. The average expression of each of the 100 pixel clusters was found and the z-score for each marker across the 100 pixel clusters was computed. All z-scores were capped at 3, such that the maximum z-score was 3 . Using these z-scored expression values, the 100 pixel clusters were meta-clustered using consensus hierarchical clustering into 12 metaclusters. Next, by applying the segmentation masks that delineate the boundaries of all cells in

194 the images, we counted the number of each of the 12 pixel clusters in each cell. This resulted in 195 a pixel cluster by cell count table. These counts were then normalized by cell size. Using these

196 frequency measurements as the feature vector, the cells were clustered using FlowSOM into 100

197 cell clusters. Similarly to the pixel clusters, the average expression of each of the 100 cell clusters

198 was found and the $z$-score was computed. All z-scores were capped at 3 , such that the maximum

199 z-score was 3 . Using these z-scored values, the 100 cell clusters were meta-clustered using

200 consensus hierarchical clustering into 10 cell meta-clusters. Each of the cell meta-clusters was

201 then manually annotated with its cell phenotype by assessing marker expression, resulting in a 202 total of eight cell types: B cells, T cells, dendritic cells, macrophages, NK cells, fibroblasts, 203 endothelial cells, and epithelial cells. To assess the concordance of cell phenotyping between 204 serial sections of the same tissue core, we quantified the number of each cell type in each image, 205 then calculated the average Spearman correlation of these frequencies between replicate images 206 of each core.

208 Results

209 Experimental design for MIBI-TOF replicate and IHC concordance

210 To assess the reproducibility of $\mathrm{MIBI}$ as well as benchmark against single-plex 211 chromogenic IHC, we took 13 serial sections from a TMA constructed for this study (see Methods) 212 that included disease-free controls and multiple types of carcinomas, sarcomas, and central 213 nervous system lesions (Figure 1). Here, every other slide was stained and processed for either 214 MIBI-TOF analysis or single-plex chromogenic IHC for the indicated target, in addition to slides 
215 for H\&E and unstained tissue control. To control for confounding factors due to batch effects

216 resulting from day of tissue staining and imaging, the order that each TMA was stained and

217 imaged were randomized with respect to serial sectioning order. Additionally, the order that each

218 tissue core was acquired for each TMA was randomized as well (Table 1).

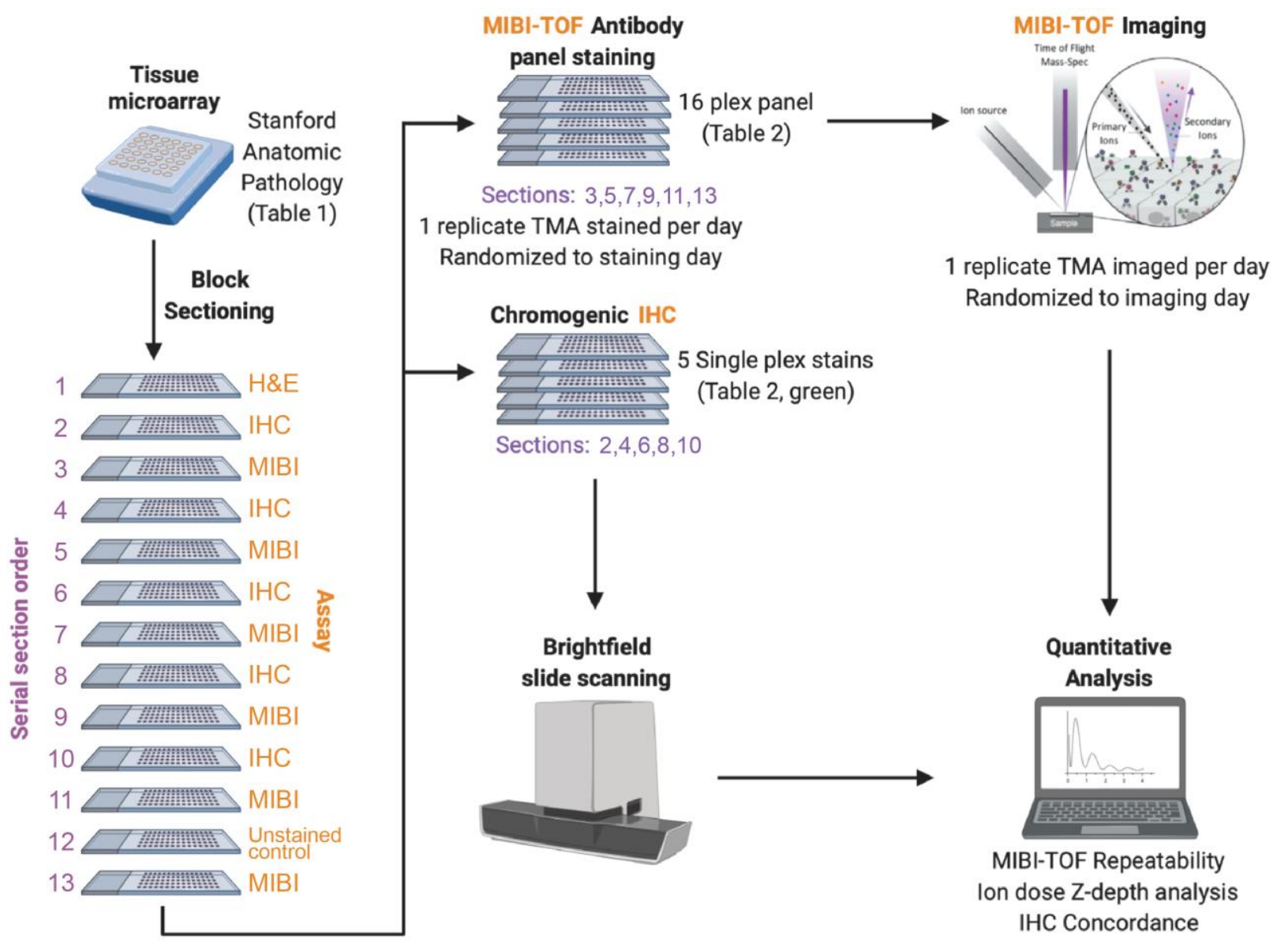

Figure 1: MIBI-TOF validation overview. A tissue microarray (TMA) was constructed using human FFPE tissue blocks, and the TMA was serially sectioned for analysis by single-plex chromogenic IHC and MIBI-TOF. The order that each serial section was stained and imaged was randomized. We then analyzed the concordance between IHC and MIBI-TOF, as well as assessed reproducibility of MIBITOF between serial sections.

MIBI-TOF replicate concordance

For MIBI-TOF, 21 tissue cores in six replicate TMAs were stained with a 16-plex antibody

222 panel and analyzed for a total of 126 MIBI-TOF images (Table 2). Instrument stability was 223 assessed prior to data acquisition using a molybdenum foil standard, for which we know the 
224 expected range of ion counts. Primary ion current and mass resolution varied insignificantly over

225 the course of the study (SD = 7\% and 1.8\%, respectively, Supplementary Figure $1 \mathrm{~A}, \mathrm{~B})$. Minor 226 variations in ion detector sensitivity $(S D=22 \%)$ were within historical norms and were not found 227 to impact subsequent quantitative comparisons (Supplementary Figure 1C). Fields in each tissue 228 core were manually co-registered with respect to a slide scan of a reference H\&E serial section, 229 which showed high concordance of histological features (Supplementary Figure 2). High quality 230 MIBI-TOF imaging data was obtained for all targets in the antibody staining panel in all six TMA 231 slides. Manual evaluation of each marker demonstrated robust and specific staining to the target 232 cells of interest in both normal and disease tissue cores (Figure 2).

Assay reproducibility was assessed by comparing marker intensity and abundance in each

234 TMA replicate. An example of co-registered fields-of-view (FOVs) from 6 serial sections of a representative tissue core is shown in Figure 3A, illustrating the high degree of reproducibility between the replicate sections. PPP values for CD3, a marker for T cells, and CD20, a marker for

237 B cells, in the fields for this tissue core were $22.6 \% \pm 2.5$ and $20.6 \% \pm 8.1$ (mean \pm SD), respectively. The significantly higher standard deviation observed with CD20 is attributable to section-to-section changes in the area occupied by two germinal centers (marked by expression of the B cell marker CD20) as sections were taken from the tissue core.

242 (PPP) for each tissue core in each TMA replicate (see Methods) were used to determine the 243 degree of concordance with respect to the average across all six replicates. For MPI, which 244 quantifies the average marker intensity in positive pixels, the resultant slope $(m)$ and coefficient 245 of determination $\left(R^{2}\right)$ values for all markers in all six replicates were close to 1 , with a $m$ of $0.96 \pm$ 2460.14 and a $R^{2}$ of $0.94 \pm 0.04$ (mean \pm SD, Figure 3B-D). MPI captures marker intensity, thus $m$ 247 and $R^{2}$ values close to 1 indicate that when a marker is present, the brightness of the marker is 248 highly concordant across replicates. Similarly, for PPP, which quantifies the total number of pixels 249 that are positive for a given marker, $m$ and $R^{2}$ in all replicates and markers were also close to 1 , 


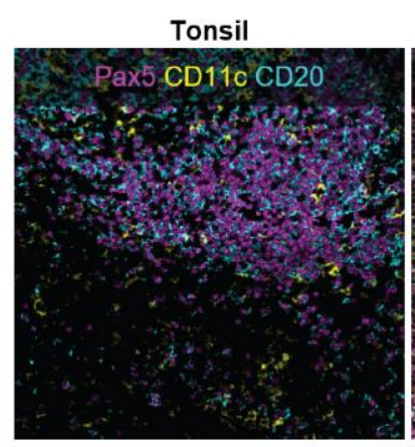

Foreign body

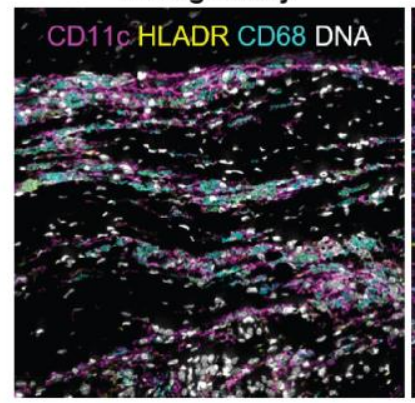

Pleomorphic sarcoma

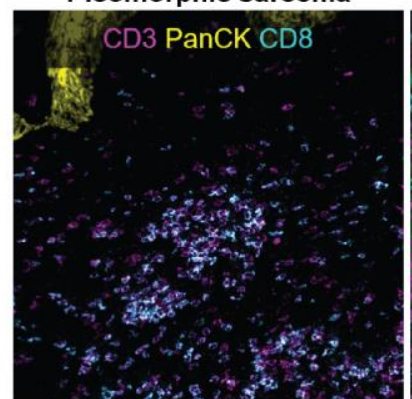

Squamous cell carcinoma

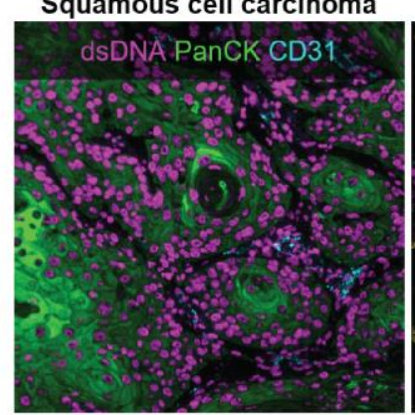

Lymph node

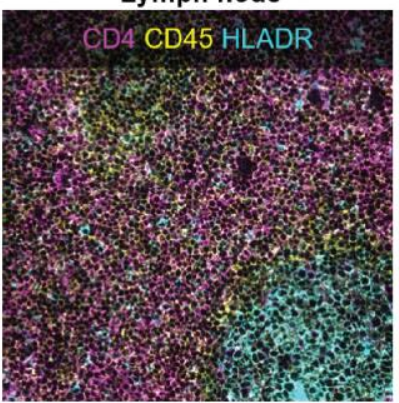

Salivary cystadenoma

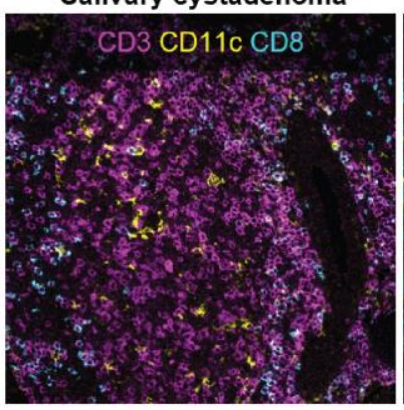

Breast ductal carcinoma

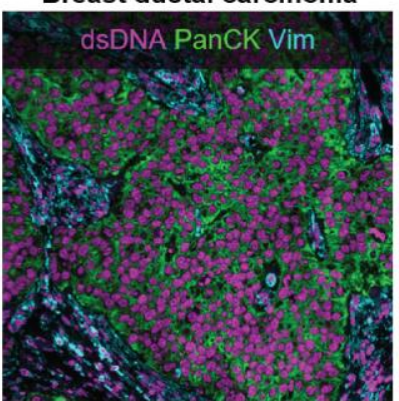

Colon adenocarcinoma

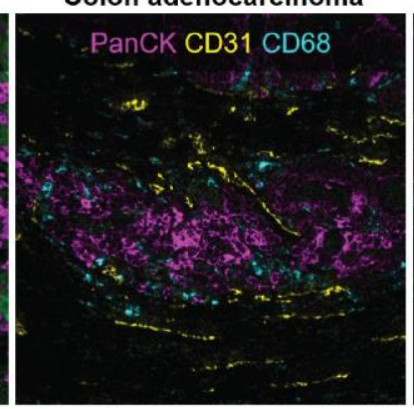

Spleen

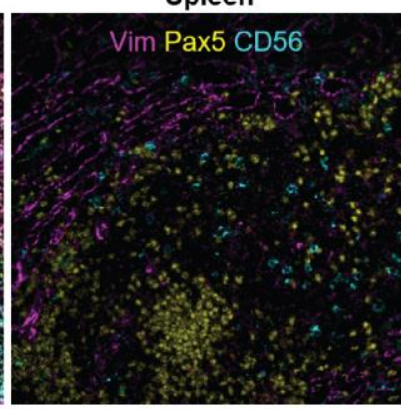

Myxofibrosarcoma

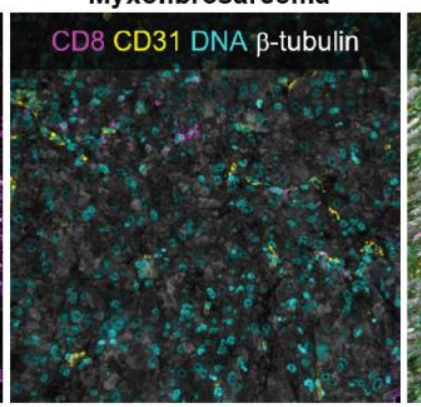

Breast ductal carcinoma

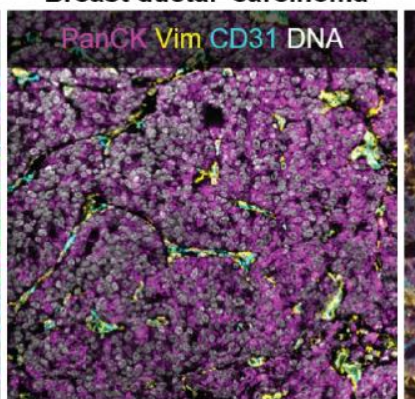

Colon adenocarcinoma

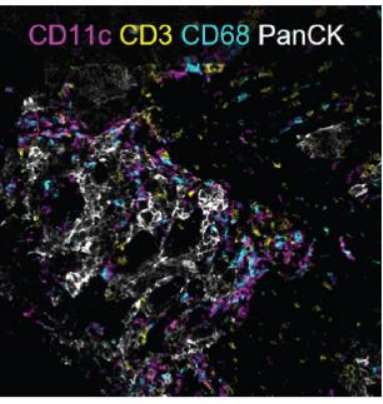

Thymus

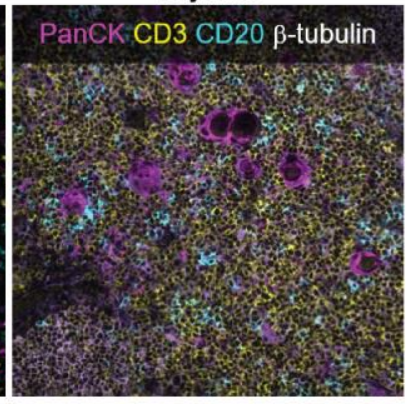

Leiomyosarcoma

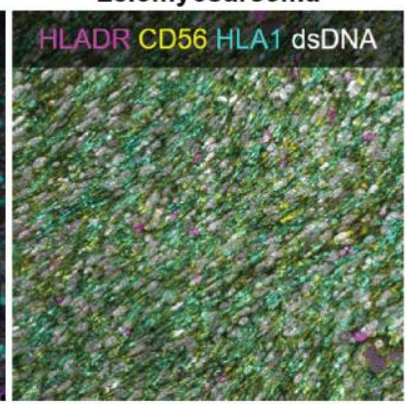

Prostate adenocarcinoma

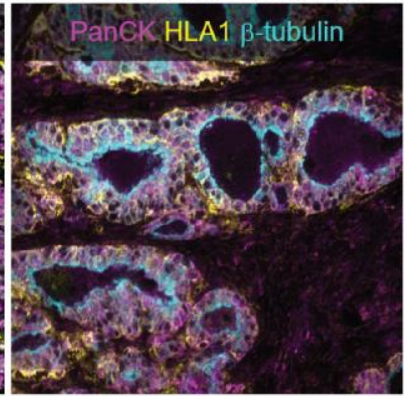

Colon adenocarcinoma

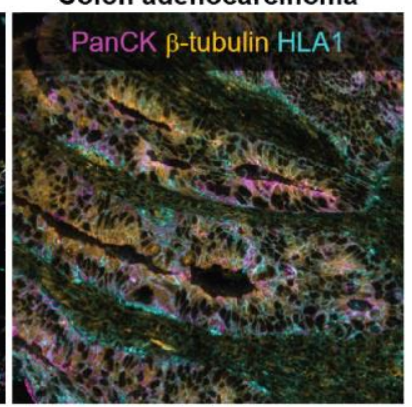

Figure 2: Representative MIBI-TOF imaging data. High-quality MIBI-TOF imaging data was attained for 16 markers for all replicate TMA slides. Representative images of the various tissue types in the TMA are shown here.

250 with a $m$ of $0.98 \pm 0.15$ and a $R^{2}$ of $0.95 \pm 0.04$ (mean $\pm S D$, Supplementary Figure 3 ). PPP $m$

251 and $R^{2}$ values close to 1 indicate that the number of positive pixels across replicates of the same

252 core is consistent. Taken together, these two metrics demonstrate that MIBI-TOF images of serial 
254 concordant, lending evidence for the reproducible nature of MIBI-TOF.
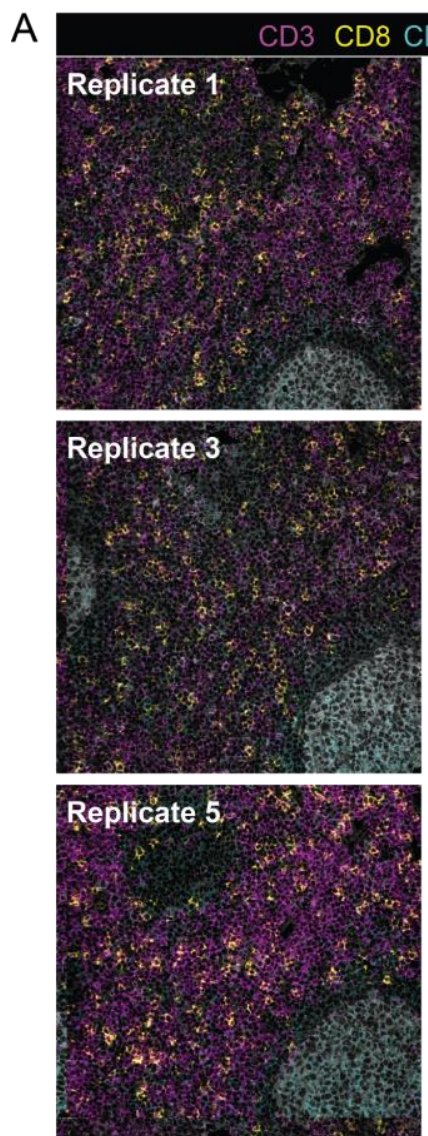
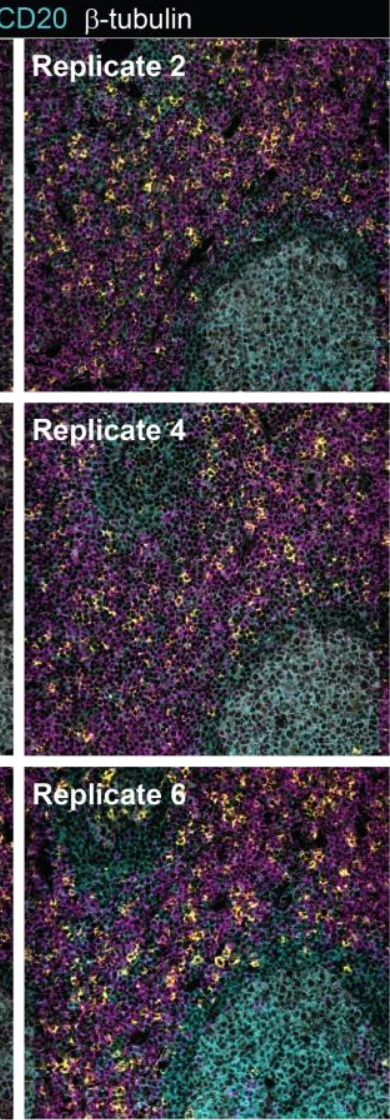

B

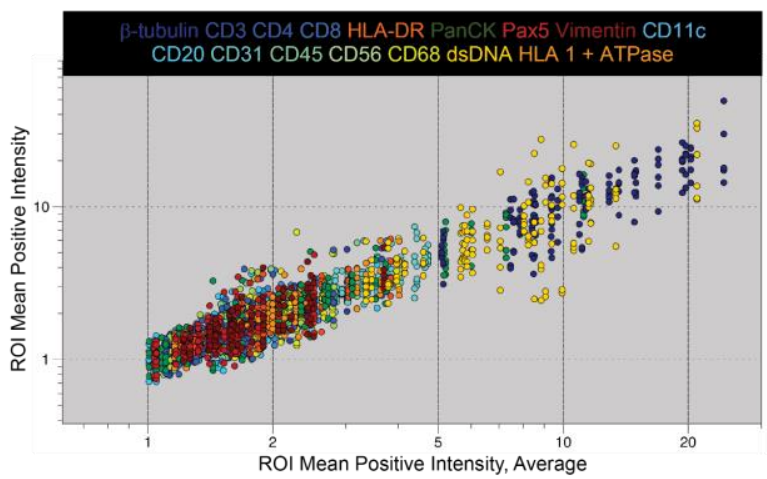

C
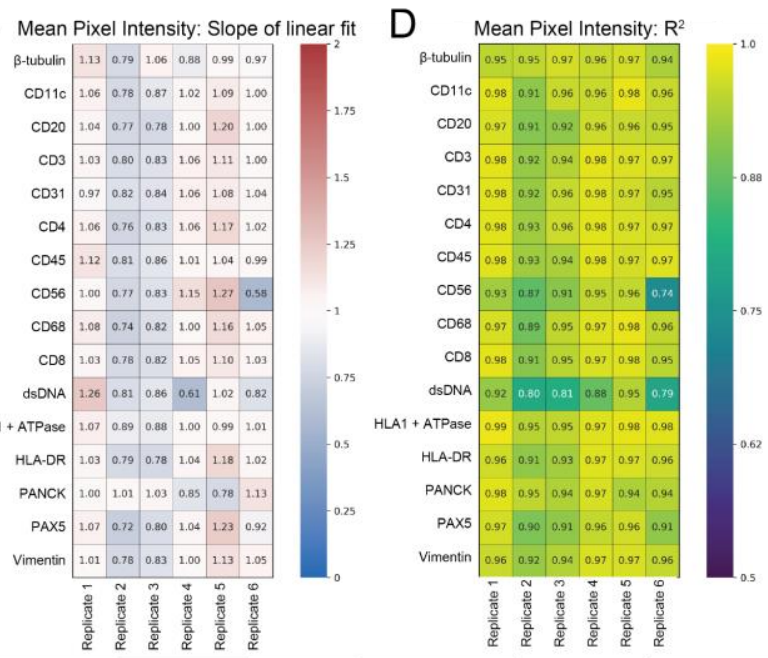

Figure 3: Concordance of Mean Pixel Intensity by MIBI-TOF. (A) Representative MIBI-TOF replicate images from serial sections of the same TMA core of lymph node tissue. (B) Plot of Mean Positive Intensity (MPI) of each FOV vs. the average MPI of all FOVs of the same TMA core. Each color represents a different marker. We performed least squares linear regression of the MPI of all six replicates of each TMA core vs the average MPI of the core and found the slope $m(C)$ and coefficient of determination $R^{2}(\mathrm{D})$ for all 16 markers.

\section{Reproducibility of cell immunophenotypic assignments by MIBI-TOF}

An important step in the analysis of high-dimensional imaging data is the identification of

257 single cell lineages by immunophenotype in the images. Thus, we next assessed the reproducibility of single-cell annotations across the replicate images of each tissue core. Our lab has recently developed a cell segmentation algorithm that can accurately identify the location of 

approach to classify the single cells into eight cell lineages: B cells, T cells, dendritic cells, 263 macrophages, NK cells, fibroblasts, endothelial cells, and epithelial cells (Figure 4A, see 264 Methods). ${ }^{17}$ After classifying the cells in each of the images, we quantified the number of each

A

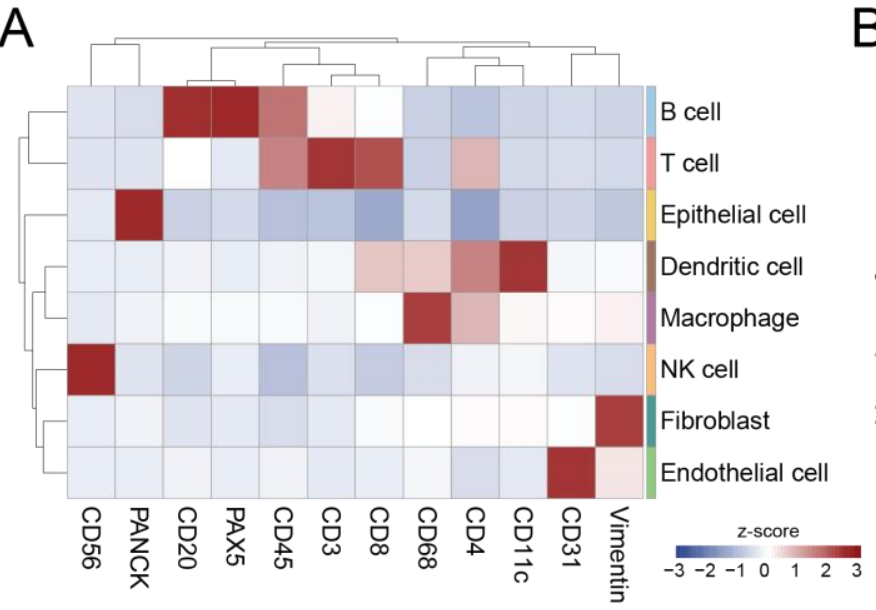

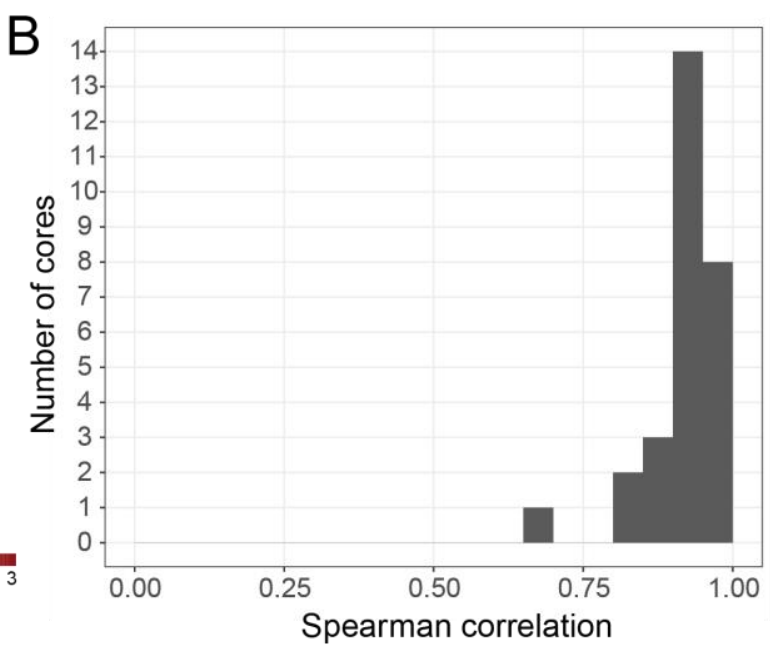

Replicate 4
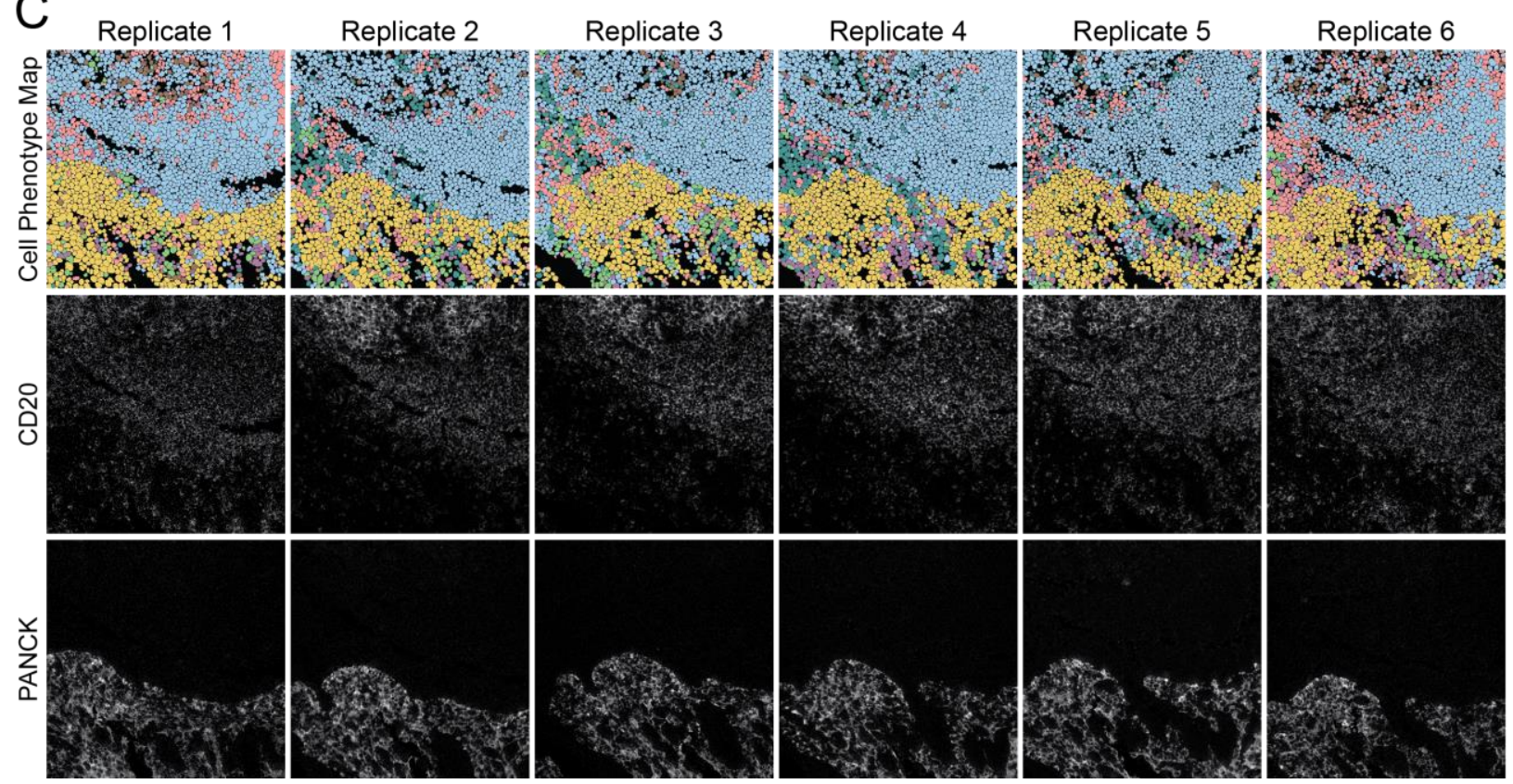

Figure 4: Reproducibility of cell phenotyping by MIBI-TOF. (A) Cells were assigned to a phenotype using an unsupervised clustering approach and manually annotated into eight major cell types. Expression values were z-scored for each marker. High z-scores are an indicator of high marker specificity. (B) The Spearman correlation between all serial sections of each TMA core using the frequency of cell types in each FOV. (C) Representative images of six serial sections of the same TMA core of tonsil tissue. Top row: Cell phenotype map colored according to the eight cell types shown in (A). Middle and bottom rows: MIBI-TOF images showing CD20 and PanCK expression. 
Spearman correlation of these frequencies between the replicate images of each tissue core

267 (Figure 4B). We expected variation in these Spearman correlation values, since the replicate 268 sections of the same tissue core are serial sections of $4 \mu \mathrm{m}$ each (with additional serial sections 269 taken for IHC concordance analysis). In total, 13 serial sections were obtained for each tissue for 270 MIBI-TOF or IHC (see Methods). Therefore, we would expect true biological differences when 271 comparing serial sections up to $48 \mu \mathrm{m}$ apart. When comparing these replicate MIBI-TOF images, 272 we observed that broad histological features were conserved, such as germinal centers or vessels

273 (Figure 3A, 4C, Supplementary Figure 4). The average Spearman correlation was high, $0.92 \pm$ 2740.06 (mean \pm SD), demonstrating that cell phenotyping, a necessary step in high-dimensional 275 image analysis, was reproducible between MIBI-TOF images of the same tissue core.

\section{Concordance of MIBI-TOF with single-plex chromogenic IHC} multiplexed imaging by MIBI-TOF can quantitatively recapitulate single-plex immunoassays.

281 Similar to the analyses described above that were used to compare MIBI-TOF data across 282 replicates, the frequency of marker positive pixels in MIBI-TOF images co-registered with single283 plex chromogenic IHC stains were compared by linear regression across all 21 tissue cores. 284 Because we alternated recuts for MIBI-TOF and IHC, we could compare MIBI-TOF and IHC on 285 adjacent serial sections. Single-plex chromogenic IHC images were acquired for five targets: 286 CD3, CD8, Pax5, PanCK, and CD68. Marker positivity by single-plex IHC for CD3, CD8, Pax5, 287 PanCK, and CD68 in adjacent serial sections were compared with the respective values found by 288 MIBI-TOF using linear regression (Figure 5, Supplementary Figure 5). For all five targets, 289 concordance of MIBI-TOF and IHC was high, with an $R^{2}$ of $0.85 \pm 0.08$ (mean $\pm \mathrm{SD}$, all $R^{2}>0.7$ ), 290 demonstrating that MIBI-TOF data is consistent with single-plex chromogenic IHC data. 

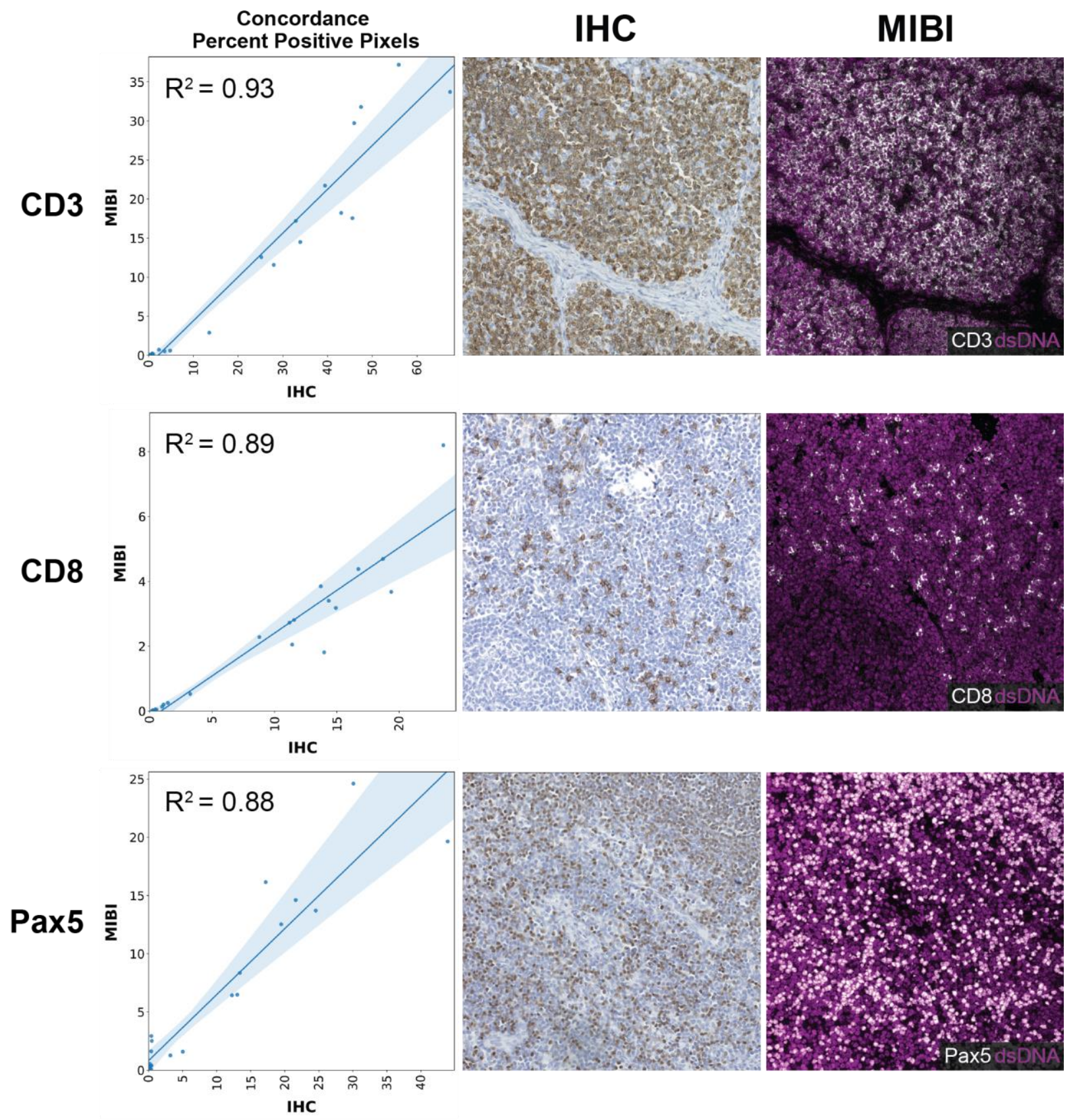

Figure 5: Concordance of MIBI-TOF with single-plex chromogenic IHC. Representative images of the comparison of MIBI-TOF images co-registered with single-plex chromogenic IHC stains. Each data point represents the PPP by MIBI-TOF and IHC for a single tissue core. Shaded area represents 95\% confidence interval. Additional markers are shown in Supplementary Figure 5.

\section{Discussion}

In this study, we assessed the reproducibility of MIBI-TOF by evaluating assay

294 concordance on six serial sections from a TMA, including disease-free tonsil, lymph node, thymus, 
and spleen, in addition to multiple types of carcinomas, sarcomas, and central nervous system lesions. Importantly, each serial section was stained independently on six separate days with a 16-marker staining panel and subsequently analyzed on six different days. 21 tissue cores were analyzed in each serial section for a total of 126 MIBI-TOF images. The staining intensity and frequency of positive pixels for each serial section (MPI and PPP, respectively) were compared against average values across all runs using least squares linear regression. In all comparisons, concordance between serial sections of the same tissue core was high, with $m$ of $0.96 \pm 0.14$ and $R^{2}$ of $0.94 \pm 0.04$ when assessing MPI and $m$ of $0.98 \pm 0.15$ and $R^{2}$ of $0.95 \pm 0.04$ when assessing PPP (mean \pm SD). The staining pattern of each marker was manually inspected to confirm, where relevant, appropriate subcellular localization and histologic distribution. For example, CD20 staining in positive regions of tonsil and lymph node in all replicates was verified to be membranelocalized and enriched in follicles. Furthermore, we assessed the reproducibility of cell phenotyping across replicates and found that across replicate sections, the location and lineage of cells identified using our data analysis pipeline were highly concordant, with an average Spearman correlation of cell type frequencies between replicates of $0.92 \pm 0.06$ (mean \pm SD). Importantly, the data analysis pipeline used to go from MIBI-TOF images to cell phenotypes was nearly fully automated. Cell segmentation and clustering were fully automated with no user input and only minimal user intervention was needed when labelling cell clusters with cell annotations, demonstrating that the biological annotation of MIBI-TOF images is robust without the need for

314 significant researcher intervention. In addition, we assessed concordance of PPP by MIBI-TOF

315 for five markers with single-plex IHC and found that MIBI-TOF and single-plex IHC were highly 316 concordant with an average $R^{2}$ of 0.85 . Taken together, the combination of consistent staining, 317 imaging, and feature extraction illustrates the highly reproducible nature of MIBI-TOF.

318 One limitation of validation studies involving serial tissue sections is that exact biological 319 replicates cannot be obtained since each tissue section is somewhat different and is not a 320 homogenized bulk sample. In this study, since the tissue sections are $4 \mu \mathrm{m}$ each and a total of 
13 serial sections were acquired, the first and last tissue section were separated axially by $48 \mu \mathrm{m}$. Also, when balancing assay replication versus concordance with an orthogonal assay, like singleplex IHC here, one needs to prioritize what physically adjacent slides are used for. In this case, we selected physically adjacent slides for benchmarking single-plex IHC since any one marker's distribution would be heavily dependent on cell composition. Lymphocytes that are approximately $\sim 10 \mu \mathrm{m}$ would be best preserved in adjacent sections. Although there will be true biological differences in this volume of tissue, this study represents the next best situation, in which we compared adjacent serial sections as a proxy for true replicates. Furthermore, this was a singlesite study, in which all staining and imaging was performed in our laboratory. We are currently planning a multi-site study to assess inter-institutional differences in instrument performance and tissue staining. Still, we believe the overall metrics for benchmarking outlined here will be sufficient to assess these future efforts.

This study was performed as a part of the Cancer Immune Monitoring and Analysis

334 Centers Cancer Immunology Data Commons (CIMAC-CIDC) network ${ }^{22}$, which is a NCI Cancer Moonshots initiative to provide the technology and expertise for immunotherapy clinical trials. Multiplexed tissue imaging is an integral assay for fully characterizing the tumor immune

337 microenvironment, which requires the simultaneous profiling of multiple tumor and immune cell

338 types. MIBI-TOF can routinely provide quantitative, multiplexed imaging data that is back 339 compatible with archival FFPE tissue and conventional anatomic pathology workflows. Technical 340 innovations in reagents and instrumentation will further increase the throughput and multiplexing 341 ability of MIBI-TOF. In the future, we envision that MIBI-TOF will not only be used for reproducible

342 basic science research, but also enable the adoption of quantitative spatial signatures in the clinic

343 for more accurate diagnosis and therapeutic selection. At the same time, as efforts to develop

344 other IHC-centric spatial imaging technologies evolve, the analytical framework and approach laid 345 out in this study should serve as a guide for their assessment. 


\section{Data Availability}

348 The datasets generated and/or analyzed during the current study are available in Zenodo:

349 10.5281/zenodo.5542727.

350

351 Acknowledgements

352 The authors thank Sushama Varma and Matt van de Rijn for creating the tissue microarray for 353 this study.

354

\section{Conflict of Interest}

356 M.A. and S.C.B. are inventors on patents related to MIBI technology. M.A. and S.C.B. are 357 consultants, board members and shareholders in lonpath Inc.

\section{Author Contributions}

360 M.B. performed experiments and imaging. A.K., A.K., and C.C.L. performed data analysis. C.C.L. 361 and M.A. wrote the manuscript. S.C.B., R.K., and S.M.H. reviewed the manuscript. S.C.B. and 362 M.A. supervised the work.

363

\section{Funding}

365 This work was supported by DOD EOH W81XWH2110143, 1-DP5-864 OD019822, 366 1R01AG056287, 1R01AG057915, R01AG068279, 1UH3CA246633, 1U24CA224309, and the 367 Bill and Melinda Gates Foundation. 


\section{References}

1. Levenson, R. M., Borowsky, A. D. \& Angelo, M. Immunohistochemistry and mass spectrometry for highly multiplexed cellular molecular imaging. Lab Invest 95, 397-405 (2015).

2. Matos, L. L. de, Trufelli, D. C., de Matos, M. G. L. \& da Silva Pinhal, M. A. Immunohistochemistry as an important tool in biomarkers detection and clinical practice. Biomark Insights 5, 9-20 (2010).

3. Chlipala, E. A. et al. An Image Analysis Solution For Quantification and Determination of Immunohistochemistry Staining Reproducibility. Appl Immunohistochem Mol Morphol 28, 428436 (2020).

4. Thunnissen, E. et al. The Use of Immunohistochemistry Improves the Diagnosis of Small Cell Lung Cancer and Its Differential Diagnosis. An International Reproducibility Study in a Demanding Set of Cases. Journal of Thoracic Oncology 12, 334-346 (2017).

5. Yatabe, Y. et al. Best Practices Recommendations for Diagnostic Immunohistochemistry in Lung Cancer. Journal of Thoracic Oncology 14, 377-407 (2019).

6. Zaha, D. C. Significance of immunohistochemistry in breast cancer. World J Clin Oncol 5, 382392 (2014).

7. McCabe, A., Dolled-Filhart, M., Camp, R. L. \& Rimm, D. L. Automated quantitative analysis (AQUA) of in situ protein expression, antibody concentration, and prognosis. J Nat/ Cancer Inst 97, 1808-1815 (2005).

8. Taube, J. M. et al. The Society for Immunotherapy of Cancer statement on best practices for multiplex immunohistochemistry (IHC) and immunofluorescence (IF) staining and validation. $J$ Immunother Cancer 8, e000155 (2020).

9. Zimak, J., Schweller, R. M., Duose, D. Y., Hittelman, W. N. \& Diehl, M. R. Programming in situ immunofluorescence intensities through interchangeable reactions of dynamic DNA complexes. Chembiochem 13, 2722-2728 (2012). 
394 10. Neher, R. \& Neher, E. Optimizing imaging parameters for the separation of multiple labels 395 in a fluorescence image. J Microsc 213, 46-62 (2004).

396 11. Patel, S. S. \& Rodig, S. J. Overview of Tissue Imaging Methods. in Biomarkers for 397 Immunotherapy of Cancer: Methods and Protocols (eds. Thurin, M., Cesano, A. \& Marincola, 398 F. M.) 455-465 (Springer New York, 2020). doi:10.1007/978-1-4939-9773-2_21.

399 12. Binnewies, M. et al. Understanding the tumor immune microenvironment (TIME) for 400 effective therapy. Nat Med 24, 541-550 (2018).

401 13. Bodenmiller, B. Multiplexed Epitope-Based Tissue Imaging for Discovery and Healthcare 402 Applications. Cell Systems 2, 225-238 (2016).

403 14. Angelo, M. et al. Multiplexed ion beam imaging of human breast tumors. Nat Med 20, 436$404442(2014)$.

405 15. Keren, L. et al. MIBI-TOF: A multiplexed imaging platform relates cellular phenotypes and 406 tissue structure. Sci Adv 5, eaax5851 (2019).

407 16. Keren, L. et al. A Structured Tumor-Immune Microenvironment in Triple Negative Breast 408 Cancer Revealed by Multiplexed Ion Beam Imaging. Cell 174, 1373-1387.e19 (2018).

409 17. Risom, T. et al. Transition to invasive breast cancer is associated with progressive 410 changes in the structure and composition of tumor stroma. bioRxiv 2021.01.05.425362 (2021) 411 doi:10.1101/2021.01.05.425362.

412 18. McCaffrey, E. F. et al. Multiplexed imaging of human tuberculosis granulomas uncovers 413 immunoregulatory features conserved across tissue and blood. bioRxiv 2020.06.08.140426 $414 \quad$ (2020) doi:10.1101/2020.06.08.140426.

415 19. Goltsev, Y. et al. Deep Profiling of Mouse Splenic Architecture with CODEX Multiplexed $416 \quad$ Imaging. Cell 174, 968-981.e15 (2018).

417 20. Schulz, D. et al. Simultaneous Multiplexed Imaging of mRNA and Proteins with Subcellular 418 Resolution in Breast Cancer Tissue Samples by Mass Cytometry. Cell Syst 6, 25-36.e5 (2018). 
419 21. Giesen, C. et al. Highly multiplexed imaging of tumor tissues with subcellular resolution by 420 mass cytometry. Nat Methods 11, 417-422 (2014).

421 22. Chen, H. X. et al. Network for Biomarker Immunoprofiling for Cancer Immunotherapy: 422 Cancer Immune Monitoring and Analysis Centers and Cancer Immunologic Data Commons 423 (CIMAC-CIDC). Clin Cancer Res 27, 5038-5048 (2021).

424 23. Baranski, A. et al. MAUI (MBI Analysis User Interface)—An image processing pipeline for 425 Multiplexed Mass Based Imaging. PLOS Computational Biology 17, e1008887 (2021).

426 24. Greenwald, N. F. et al. Whole-cell segmentation of tissue images with human-level 427 performance using large-scale data annotation and deep learning. bioRxiv 2021.03.01.431313 $428 \quad$ (2021) doi:10.1101/2021.03.01.431313.

429 25. Van Gassen, S. et al. FlowSOM: Using self-organizing maps for visualization and 430 interpretation of cytometry data. Cytometry A 87, 636-645 (2015). 